\title{
Early detection of acute coronary syndromes and risk stratification by multimarker analysis
}

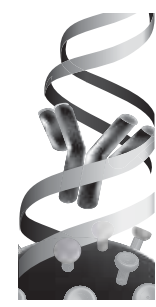

\author{
Alan HB Wu \\ University of California, \\ $D$ epartment of Laboratory \\ $M$ edicine, San Francisco, \\ CA, USA \\ Tel.: +1 415206 3540; \\ Fax: +1 415206 3045; \\ E-mail: wualan@ \\ labmed2.ucsf.edu
}

\begin{abstract}
Cardiac troponin is the standard biomarker for the diagnosis of acute myocardial infarction (AMI) and risk stratification for short-term adverse cardiac events (death, AMI or need for revascularization). Unfortunately, the concentration of troponin in blood is normal in AMI patients who present early after the onset of symptoms. As such, there is active research being conducted in finding early markers of AMI and risk stratification. Despite years of testing dozens of candidates, no single test has had the necessary clinical sensitivity and specificity for this indication. Therefore, many researchers have advocated multimarker testing. There are two approaches that have been taken for discovering new markers. The proteomic approach involves focusing on the differences in the biochemical signatures between the tissues or biological fluids of normal compared with diseased individuals. Specific biochemical targets are not preselected. The pathophysiologic approach involves combining biomarkers that indicate a particular pathway or event known to be involved in the disease process. In both approaches, some bioinformatic algorithm will be necessary in order to combine the information provided by the individual tests. Representative approaches include the Multimarker Index ${ }^{\mathrm{Tm}}$, classification and regression tree analysis and neural networks.
\end{abstract}

Cardiovascular disease (CVD) continues to be the leading cause of morbidity and mortality in the USA and throughout the western world [101]. According to the American Heart Association, approximately 64.4 million Americans have CVD, which accounts for $38.5 \%$ of all deaths [101]. Approximately 13.2 million have coronary artery disease, 7.8 million have suffered an acute myocardial infarction (AMI) and 6.8 million have suffered angina pectoris [101]. CVD also accounts for a large number of individuals who present to the emergency department (ED) with chest pain. Approximately eight out of a total of $95 \mathrm{ED}$ admissions ( $28 \%)$ are due to patients who present with chest pain or other symptoms suggestive of CVD (Figure1). A large fraction of patients have a noncardiac source of their symptoms, such as skeletal muscle pain, pulmonary emboli, aortic dissection, blunt trauma to the chest or emphysema, amongst other factors and are ruled out for AM I. The rate of discharge is dependent on the aggressiveness of the hospital in sending patients home. The remaining patients are admitted either to a chest-pain center for rapid rule out of AMI, a hospital bed or the coronarycare unit. M ore than $50 \%$ of these patients will be ruled out for acute coronary syndromes (ACS) within the ensuing 1-3 days. Of the remaining patients, there are roughly 1.2 million who suffer AMI and another 800,000 who have unstable angina. There are also a significant number of patients who die of ACS before they appear in the ED or shortly after arrival. U sually death is caused by cardiac dysrhythmias induced by the AMI, which occurs before traditional biomarkers are released into the circulation.

\section{Pathophysiology of ACS}

Although there are many etiologies to AM I, the occlusion of a coronary artery by the erosion or rupture of an atherosclerotic lesion on a coronary artery is responsible for most cases [1]. There are many events that occur leading up to the rupture of this artery. Figure 2 illustrates the various stages of ACS. Stage A shows a clean coronary artery [2]. Atherosclerosis (stages $B$ and $C$ ) typically begins during early adulthood depending on diet and lifestyle habits. In a stable plaque (stage D ), there is a significant lesion that protrudes into the coronary artery and is filled with lipids (shown in yellow). A thick fibrous cap protects this lesion from rupture. Stage $E$ shows the development of a plaque that is vulnerable to rupture under the sheer stress of arterial blood pressure. Inflammation and leukocyte infiltration release cytokines and enzymes that degrade the collagen layers that protect the artery. AM I occurs following plaque rupture (F). The release of lipids and extracellular matrix 'gruel' stimulates platelet activation and thrombus formation. 


\section{Figure 1. Annual visits to the emergency departments in US hospitals and the incidence of acute coronary disease among those visits.}

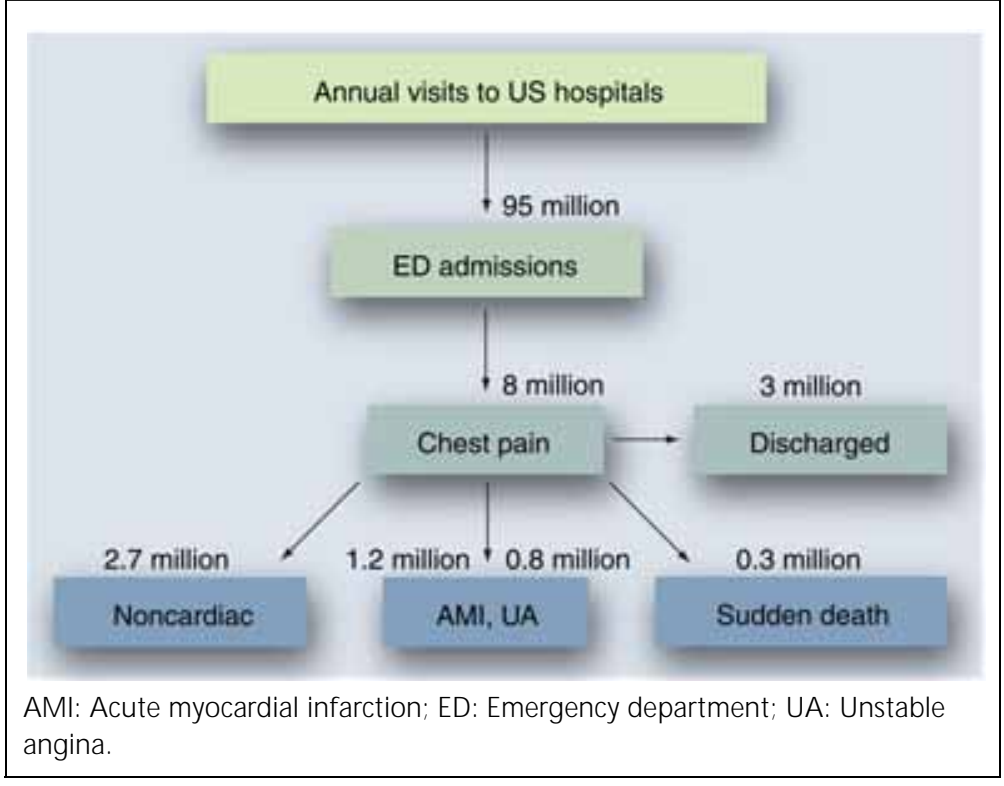

Existing biomarkers for acute myocardial infarction

Creatine kinase $(C K)$ and its $M B$ isoenzyme (CK-MB) was the mainstay for the diagnosis of AMI. CK is found in the heart, striated and smooth muscle and brain. Measurement of CK-M B has higher specificity towards cardiac injury. Troponin is today the preferred test for AM I. Cardiac troponins T ( $C T$ nT) and I (CT nl) are found in higher concentrations in the heart than $\mathrm{CK}$, and are more sensitive toward detecting myocardial cell death. Troponin is also more specific towards heart disease, as the skeletal muscle troponin isoform is distinct from the cardiac form [3]. M yoglobin is a small protein that is released earlier after AMI than troponin or CK-MB. Myoglobin is falsely increased in patients with skeletal muscle disease and renal failure and, therefore, the utilization of myoglobin has greatly diminished. Before these macromolecules can traverse the cell membrane and appear in blood, there must be irreversible myocyte necrosis. M oreover, the occlusion of a coronary artery blocks the egress of proteins directly into coronary circulation. Therefore, there is a delay from the onset of plaque rupture to the appearance of biomarkers into blood, and levels are normal during their initial ED presentation. Passage of cardiac proteins must occur through lymphatic drainage, which adds further delay to their clearance.
B-type natriuretic peptide (BN P) and N -terminal prohormone brain natriuretic peptide (NT-proBNP) are other widely used cardiac biomarkers. These peptides originate from the ventricles of the heart, are released into the blood in response to volume overload and myocardial wall stress, and are used for the diagnosis of heart failure. BN P and NT-proBN P are also released in patients with myocardial ischemia from ischemiainduced ventricular dysfunction and hypoxia. Increased concentrations of BN P/NT-proBNP are associated with an increased risk for cardiovascular death, and many studies have begun to incorporate them as part of a multimarker panel.

\section{ESC/ACC redefinition of AMI}

In 1979, the WHO defined AM I on the basis of finding two out of three equally-weighted criteria: clinical history of an ischemic presentation, unequivocal electrocardiographic (ECG) changes and serial changes in cardiac enzymes in blood [4]. W ith the W H O criteria, it was possible to diagnose AMI without finding an increase in CK-MB. With the development of troponin, the European Society of Cardiology (ESC) and the American College of Cardiology (ACC) redefined the criteria for AMI in 2000 [5]. It is now predicated on the finding of a rise and fall of a cardiac biomarker with evidence of myocardial ischemia (ischemic symptoms, ECG changes, evidence by cardiac catheterization, or pathologic changes at postmortem). Cardiac troponin was recommended as the preferred marker for AMI diagnosis, replacing CK-MB. The motivation for making these changes was based on the clinical observation that any increase in troponin was correlated with an adverse shortterm outcome (i.e., cardiac death, AM I or need for revascularization within 30 days). Approximately a third of the cases previously defined as unstable angina had increases in troponin, suggesting the presence of irreversible damage and subsequent cardiovascular risk that is similar to the rate seen for AMI. The ESC/ACC Committee recommended using a troponin cutoff at the upper 99th percentile of a healthy population, ideally using an assay with an imprecision of less than $10 \%$ at this cutoff.

\section{Clinical need for early biomarkers of acute coronary syndromes}

Although troponin is now the gold standard marker for AM I, this marker is usually normal when blood is collected within the first few hours after symptoms onset. Therefore, for a 


\section{Figure 2. Stages in the pathophysiology of acute coronary syndromes.}

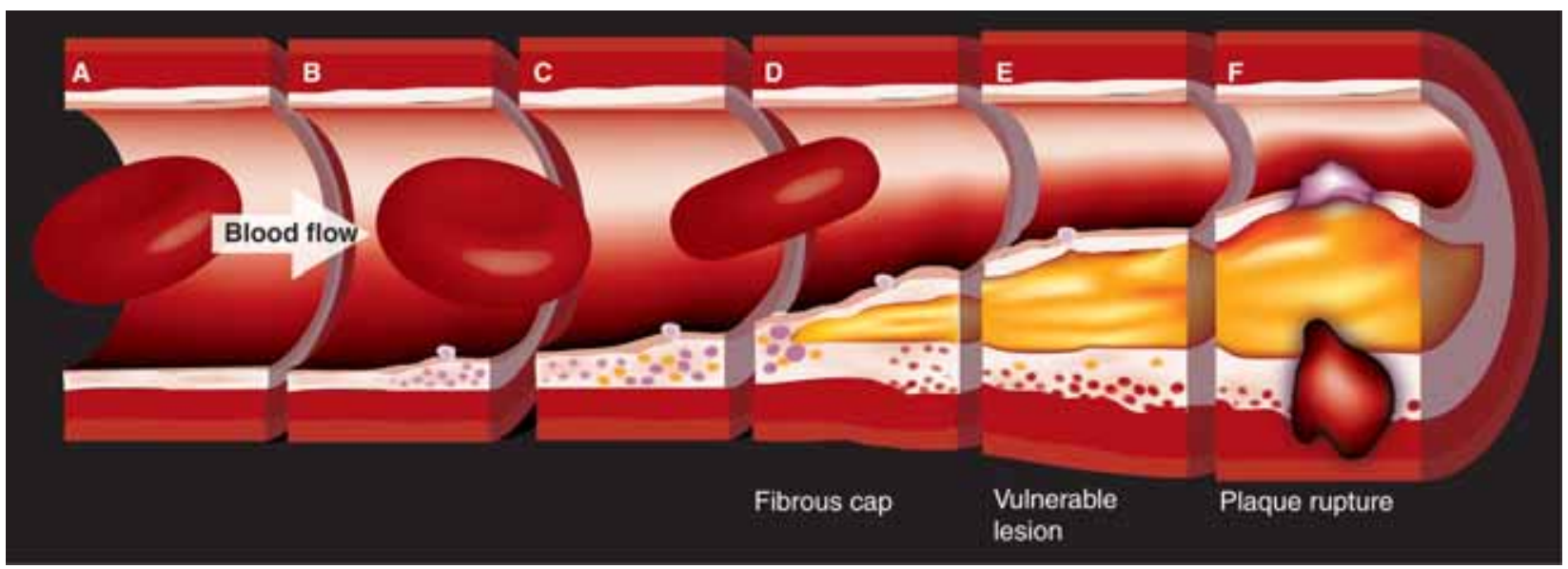

(A) Coronary artery that is largely free of plaques. (B \& C): Progressively increasing stages of plaque formation. (D) A plaque that has formed a lipid core. A thick fibrous cap protects the lipid core from the circulation. (E) A plaque that is vulnerable to rupture, characterized by a thin fibrous cap. (F) A ruptured plaque. The exposure of the lipid core to circulating blood activates the thrombosis cascade and the aggregation of platelets (not shown). Adapted and used with permission from [2].

patient who presents early, ED physicians must wait a few hours before diagnosis and treatment can be initiated. M oreover, there are substantial costs that are incurred for keeping patients in a crowded ED while a definitive diagnosis is being established. Some hospitals have opted to reduce their costs by instituting an aggressive discharge policy. Unfortunately, this will increase the rate of inadvertent discharge of patients who have AMI or will suffer one in the near term (e.g., $24 \mathrm{~h}$ ). M issed AM Is are the leading cause of malpractice lawsuits in the ED today [6].

In order to reduce hospital expenses and maintain diagnostic accuracy, many ED s have developed 'chest pain' centers of the rapid ruleout of CVD. The frequency for cardiac biomarker and ECG testing is increased. If a patient has a positive result with either test, they are immediately admitted to an appropriate hospital bed. If these tests are negative 6- $9 \mathrm{~h}$ after presentation, patients are sent for a stress test or a nuclear imaging analysis for evaluation of their cardiac status. Those with negative stress test results are unlikely to suffer a major cardiac event within the next few days and are medically cleared for discharge [7].

A patient who presents to the ED with symptoms or electrocardiographic evidence suggestive of myocardial ischemia, and has a positive troponin result in serum defines an AMI. This patient should be admitted and appropriately treated. No further laboratory testing is needed to triage this patient. While troponin is the gold standard for AM I diagnosis, there are remaining questions and clinical situations that are not answered when the results of troponin are negative. Therefore, a biomarker or a panel of tests is needed that is:

- Reliably increased at the time of ED presentation of a patient with ACS, before troponin is increased;

- Consistently negative at ED presentation of patients who are ultimately ruled out for AMI;

- Predictive of short-term cardiovascular morbidity or mortality with a negative troponin at all time points.

\section{Search for novel biomarkers of CVD} using proteomics \& metabolomics The identification of novel biomarkers for CVD detection is a very active area of research. The analytical methodologies and ideal characteristics of a biomarker have recently been reviewed [8]. There are two general approaches towards the search for any biomarkers for human diseases. In the proteomics and metabolomics approach, tissue and/or blood samples from diseased individuals are compared against either healthy subjects or patients who have a disorder that mimics the disease in question $[9,10]$. For example, blood or tissues from patients with early myocardial ischemia would be compared with patients who present with noncardiac chest pain. The samples are subjected to 2D electrophoresis for separation and the individual proteins or metabolites are identified by sophisticated mass spectrometric techniques. The 
proteomic/metabolomics approach does not presume any prior knowledge of the pathophysiology for the disease in question. The proteins or metabolites may not be known or characterized for this purpose.

To promote proteomics, The $\mathrm{N}$ ational $\mathrm{H}$ eart, Lung and Blood Institute assembled a proteomics working group specifically targeted towards new CVD biomarker discovery [11]. While proteomics has tremendous potential in finding new markers, the working group identified major challenges. There are many steps and expenses necessary to bring biomarker discoveries into a clinical test approved by the US FDA for use on patients. In vitro diagnostic corporations do not have budgets to conduct the trials necessary to gain clearances. $M$ any of the novel biomarker studies are therefore validated as part of substudies originally designed and funded for new cardiovascular drugs. D issemination of new assays may also be limited by patents that have been granted on specific proteins and their application to clinical practice. The N IH has begun funding multidisciplinary translational research projects to bring innovative lab tests from the "research bench to patient's bedside" [102].

There have been a few studies that used these techniques to discover new cardiac biomarkers. Using carotid endarterectomy tissue samples, $M$ artin-Ventura and colleagues used differential proteomics and reported that heat-shock protein (H SP)-27 was decreased in complicated atherosclerotic plaques [12]. H igh levels of H SP-27 may be cardioprotective by inhibition of cardiovascular inflammation and apoptosis. M arshall and colleagues examined serum from patients with AMI and compared results against healthy controls [13]. M ass spectrometric analysis showed that after AM I, the $\alpha$-chain of complement C 3 and fibrinogen are degraded to $\mathrm{C} 3 \mathrm{f}$ peptide and fibrinogen peptide $A$, respectively, which are then further degraded to smaller components by aminopeptidase to form a distinctive fingerprint pattern. 0 ther investigators have used proteomic techniques to identify early stages of heart failure, which could have a significant impact on morbidity and mortality rates if early disease detection can belinked to therapeutics to slow or prevent disease progression [14].

W hile proteins are the usual target for many proteomic disease correlations, lower molecular weight targets may be appropriate for ACS. This is because proteolytic enzymes are also released into blood that can degrade cardiac proteins. Thus, some investigators have focused on metabolomic techniques. Brindle and colleagues used nuclear magnetic resonance imaging of human serum to determine the presence and severity of coronary heart disease [15]. With a clinical specificity of more than $90 \%$, they were able to discriminate severe multivessel stenosis $(>90 \%)$ from patients with normal coronary arteries. Sabatine and colleagues also used metabolomics to identify novel biomarkers of myocardial ischemia [16]. These investigators collected blood from patients before and after exercise stress testing and identified 18 patients who had an inducible ischemia and 18 patients who were normal. Some 23 metabolites were found at higher concentrations in ischemic compared with nonischemic cases, including six members of the citric acid pathway. Kiernan and colleagues used a multiplexed mass spectrometric immunoassay technique as a tool for biomarker discovery, identification and verification [17].

The use of proteomic and/or metabolonomic techniques in routine clinical practice appears to be a few years away. The mass spectrometric instrumentation is expensive and requires specialized operator training, and is currently not amendable towards routine clinical analysis. This is particularly true for markers of myocardial ischemia, as the optimum application of these tests is in the ED, and a rapid turnaround from sample collection to analysis, would be desired. It is very likely that proteomics will enable the identification of a few useful nonredundant targets that can be assembled into an array of immunoassays. Clinical laboratory instrumentation is moving towards multimarker analysis with the development of genechip arrays and multicolored-bead analyzers [18]. When a list of analytes has been identified, it would beappropriateto collaborate or license such technologies with these manufacturers.

Novel biomarkers based on the pathophysiology of ACS

In addition to proteomics/metabolomics, the alternate approach towards biomarker discovery is to focus on the known processes that participate in the pathophysiology of CVD for the specific development of ACS; it is particularly important to focus on processes that participate in the conversion of stable atherosclerosis to the vulnerable plaque [19].

\section{Markers of inflammation \&}

plaque instability

M yocardial inflammation stimulates the recruitment of cellular and humoral elements into the 
coronary arteries. Their presence is responsible for the thinning of the fibrous cap and increasing the vulnerability of previously stable atherosclerotic lesions and they are targets for biomarker discovery.

\section{C-reactive protein}

M yocardial inflammation is a key step in the formation of unstable coronary artery lesions. Inflammation triggers the release of biochemical factors that simultaneously protect the body from foreign antigens and damages vital organs such as the heart. C-reactive protein (CRP) was the first inflammatory marker studied for CVD detection. CRP is an acute phase reactant whose concentration is grossly increased (e.g., $>100 \mathrm{mg} / \mathrm{l}$ ) in the blood of patients with systemic inflammation. In the absence of a global inflammatory process, localized myocardial inflammation can occur. Using high-sensitivity (hs) CRP assays, the finding of a minor elevation in hSCRP ( 3 to $10 \mathrm{mg} / \mathrm{l}$ ) has been associated with increased primary risk for CVD over the ensuing years [20].

While hsC RP is not a useful diagnostic test for early AM I or rule-out, it has been extensively studied as a predictor of secondary disease risk for patients with a history of ACS or those who present with acute symptoms of myocardial ischemia. Interpretation of hSCRP test results in this context, however, is complicated by the fact that AM I will stimulate an increase in CRP, there fore, an increased cutoff level greater than $10 \mathrm{mg} / \mathrm{l}$ may be necessary for secondary risk stratification. The nonspecificity of hsC RP in the acute setting has limited its application in the ED. H owever, hsCRP may be useful as a part of a multimarker panel for cardiovascular risk assessment. Sabatine and colleagues examined the combination of hSC RP, troponin and BNP on patients from two large clinical trials [21]. These investigators found that the three tests were independent predictors for death, AMI or heart failure. Relative to one marker being abnormal, there was a doubling in the death rate at 30 days for each additional marker that was increased. This study was one of the first to use a multimarker approach towards risk stratification for CVD. There was no attempt to change the cutoff concentrations of theindividual markers or use an algorithm to optimize the interpretation of results.

Placental-like growth factor

Placental-like growth factor (PIGF) is a member of a family of vascular endothelial growth factors, and is thought to be an instigator of vascular inflammation. It functions to stimulate smooth muscle growth, recruit monocytes and macrophages into coronary artery plaques and upgregulate proinflammatory cytokines such as tissue necrosis factor $\alpha$. As with hsC RP, increased concentrations of PIGF in the blood is a nonspecific indicator of myocardial inflammation. In the c7E3 Fab Anti Platelet Therapy in Unstable REfractory angina (CAPTURE) trial, increased concentrations of PIGF was associated with a higher risk for death or nonfatal $\mathrm{AMI}$ at 30 days (odds ratio [O R ]: 3.34; 95\% confidence intervals [CI]: 1.79-6.24) [22]. Using a multivariate model, these investigators found PIG F to be independent from CTnT and soluble CD 40 (SCD 40) ligand (discussed below). H owever, hsC RP was found to be a dependent variable.

\section{Myeloperoxidase}

Myeloperoxidase (M PO) is a heme-containing enzyme that is found in azurophilic granules of polymorphonuclear leukocytes. It functions to oxidize chloride to hypochlorous acid, which has bacteriacidal properties. Increased activities of M PO are found in the shoulder regions of coronary artery plaques, where M PO oxidizes lowdensity lipoprotein. It also activates matrix metalloproteinases (M M Ps) which can degrade the collagen, the protective layer of the fibrous cap, thereby making it vulnerable to rupture. M PO appears to be a marker of risk stratification for ACS. Zhang and colleagues reported an OR of 11.9 (95\% Cl: $5.5-25.5)$ [23]. In a multivariate analysis, Baldus and colleagues showed that M PO , CT nT, vascular endothelial growth factors, SCD 40 ligand and CRP were independent predictors of 6-month outcomes [24].

Pregnancy-associated plasma protein A

Pregnancy-associated plasma protein A (PAPP-A) and M M P-9 were first identified in the plasma of pregnant women. PAPP-A is an enzyme that works with other metalloproteinases, such as MMP-9 and myeloperoxidase, to degrade the fibrous cap. PAPP-A from pregnant women is bound to the proform of eosinophil major basic protein (proM BP), while that from the coronary artery is free PAPP-A [25]. Therefore, it is important for patients with ACS that the PAPP-A assays that recognizes either total or free PAPP-A are used, and not just the PAPP-A bound to proM BP [26]. Like PIGF, high concentrations of PAPP-A are associated with increased risk for short-term cardiovascular risk (O R: 2.44; 95\% Cl: 1.25-5.89) [27]. PAPP-A remained an independent variable when 


\section{Figure 3. Relationship betw een myocardial oxygen supply and demand.}

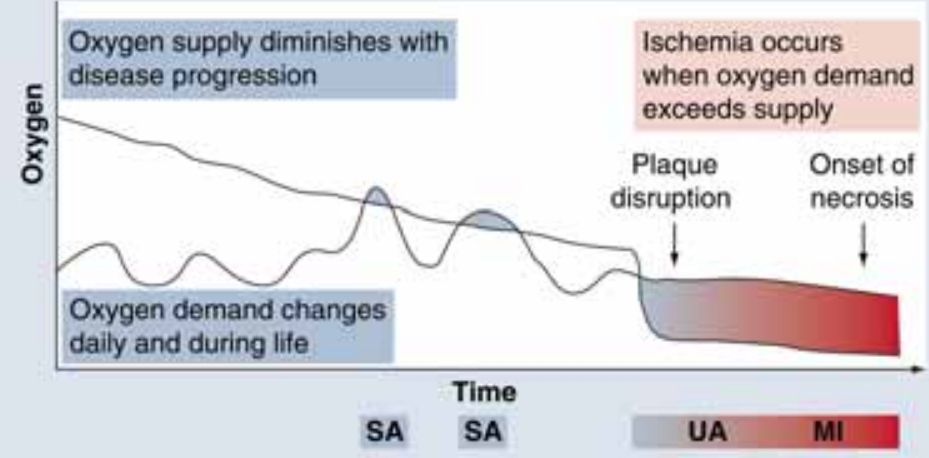

Under normal conditions, the oxygen supply greatly exceeds the demand and there are no ischemic episodes. As an individual ages, the formation of plaques reduces the volume of blood that flows through the coronary artery and the supply of oxygen. Stable angina (also termed 'demand ischemia') occurs when the demand temporarily exceeds the supply, such as during exercise, and results in chest pain for the subject. Plaque rupture results in a more dramatic and prolonged drop in blood flow due to the blockage of a coronary artery. Unstable angina is characterized by chest pain at rest and results from oxygen supply deficit for an extended period of time. The injury is initially reversible, but if the artery is not opened within a few hours, the individual has irreversible heart damage and suffers a myocardial infarction. This is characterized by the release of cardiac troponin.

MI: M yocardial infarction; SA: Stable angina; UA: Unstable angina. combined with troponin, SCD 40 ligand, and vas cular endothelial growth factors. hsCRP dropped out of significance in a stepwise multivariate regression analysis model.

Markers of plaque rupture, thrombosis \& myocardial ischemia

The rupture of a coronary artery plaque initiates a cascade that stimulates platelet aggregation, thrombus formation, reduced coronary artery blood flow leading to myocardial ischemia and, finally, myocardial cell death. The total occlusion of a coronary artery is an ST-elevation M I (STEM I) and causes a large release of troponin. The non-ST-elevation MI (nSTEM I) results in a partially occluded artery, and generally has little or no myocardial damage or troponin release. H owever, any coronary artery plaque rupture is a harbinger of adverse cardiac outcomes, and biomarkers are needed to denote the presence of this event, even if troponin remains consistently negative.

\section{CD40 ligand}

CD 40 is a receptor molecule found on monocytes, macrophages, endothelial cells and platelets [28]. Ligands for CD 40 consist of membrane bound and SCD 40 components. Activation of platelets by agonists such as adenosine monophosphate, collagen and arachidonic acid results in the upregulation and subsequent release of the soluble form into the circulation. SCD 40 ligand can then activate endothelial cells and stimulate an inflammatory response within a coronary artery plaque. Patients in the highest quartile of SCD 40 ligands were associated with adverse outcomes at 10 months [29]. Given the role of the SCD 40 ligand in platelet activation, blood levels of SCD 40 ligand may al so be important in selecting patients who would benefit most from antiplaletet drug therapies. H eeschen and colleagues demonstrated that patients with increased SCD 40 ligand levels had a lower incidence of death or AM I when treated with the glycoprotein $\mathrm{IIb} / \mathrm{Illa}$ inhibitor abciximab than patients with high SCD 40 ligand levels treated with placebo [30]. There was no difference in morbidity or mortality when abciximab was given to patients with a low SCD 40 ligand level. Although platelet activation can occur in many noncardiac diseases, a biomarker of platelet activation will likely provide independent information to a panel of markers that address inflammation and plaque instability. This is particularly true for nSTEM I, as platelets play a bigger role in subocclusive blood clots than occlusive clots.

Whole-blood \& plasma-blood choline

Choline and phosphatidic acid are released after the simulation of phosphlipase $D$ that is found on platelets, leukocytes and smooth muscles. Like SCD 40 ligand, the appearance of choline indicates a combination of plaque destabilization and cell activation. This marker is different from the other markers discussed in that it is not a protein, but a low-molecular-weight metabolite. It is unlikely that a traditional immunoassay can be developed to measure whole-blood or serum choline. The choline that is released from phospholipase D activation appears in erythrocytes, but not plasma. Increased whole-blood choline is associated with increased short-term cardiovascular death or cardiac arrest risk (O R: 6.0; 95\% Cl: 1.85-19.49) [31]. An interesting additional benefit is the observation that choline is released into the blood and plasma of patients with severe lifethreatening arrhythmias, presumably due to tissue ischemia [2]. The measurement of both red-cell and plasma choline enables a differentiation between plaque destabilization (whole-blood choline only) from arrhythmias (both whole-blood and plasma choline). 
Figure 4. Multimarker approach for early diagnosis of acute coronary syndromes based on plaque rupture as the major etiological event.

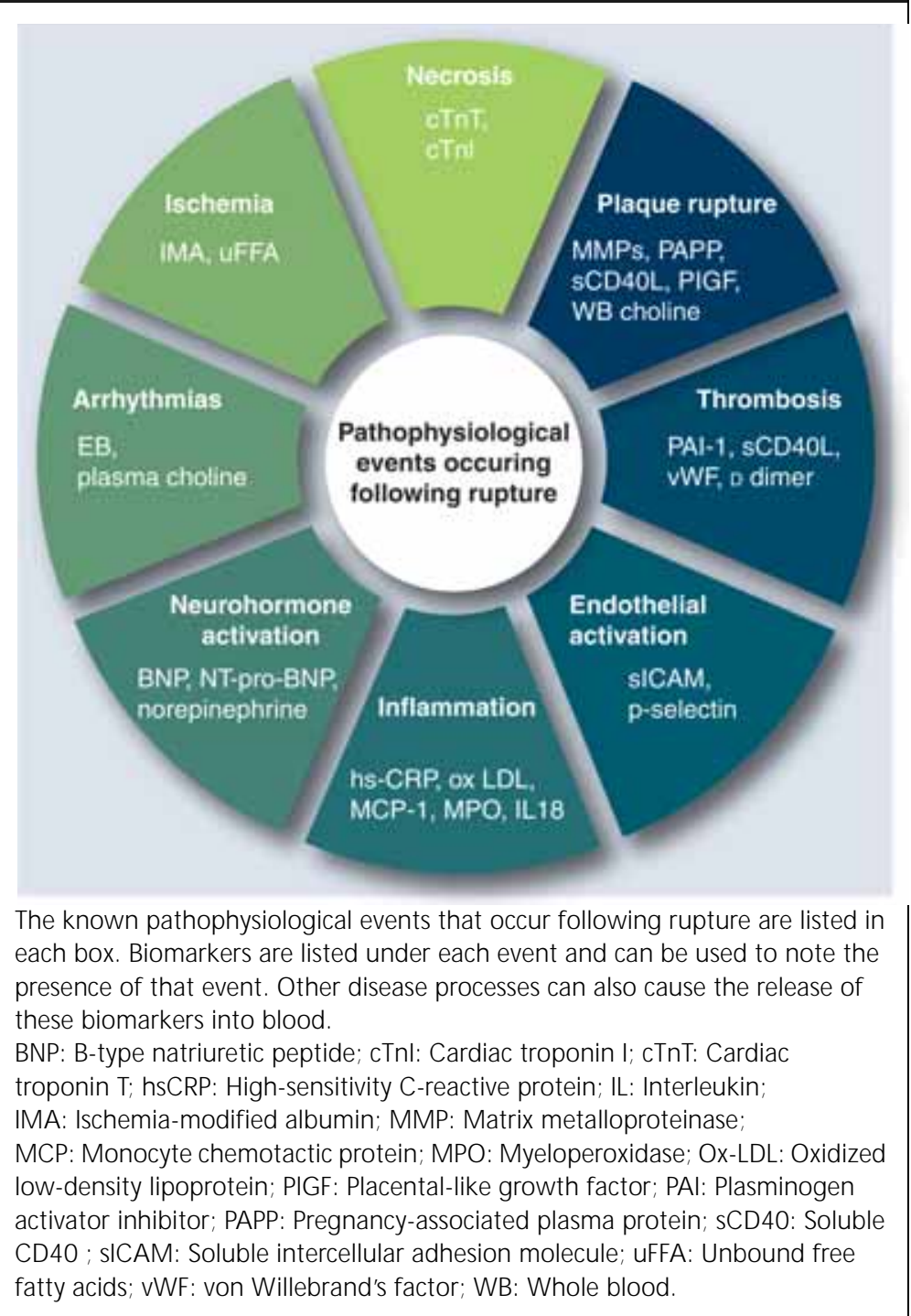

Ischemia-modified albumin

Myocardial ischemia preceeds irreversible cell death in patients with AMI. Ischemia results from a deficit between oxygen delivery and demand (Figure3). A transient increase in the demand for oxygen, such as in stress or exercise, leads to stable angina, and is characterized by chest pain. A prolonged drop in the delivery of oxygen occurs when there is plaque rupture that causes unstable angina and AM I if the blockage is prolonged. Spontaneous reperfusion or therapeutic revascularization of the coronary artery is necessary to restore coronary artery oxygen delivery. The presence of ischemia leads to the production of hydrogen peroxide and other free radicals. An alteration in the $\mathrm{N}$-terminus of albumin caused by free radical damages causes this protein to lose its ability to bind to free heavy metals. The Ischemia Modified Albu$\min ^{T M}$ (IMA) test measures the decrease in cobalt binding in patients with ischemia.

There have been many studies that have examined the clinical utility of the IM A test for patient presenting to the ED with chest pain. $A$ meta analysis showed that when the IM A test is used alone, it had a negative predictive value of $91 \%$ for ruling out ACS [32]. When this test is used in a multivariate analysis in combination with troponin and ECG, the negative predictive value increased to $97 \%$. The major disadvantage of the IMA test is the poor clinical specificity. 0 ther etiologies can cause an increase in IM A such as stroke and gastrointestinal ischemia. Therefore, it is likely that this test will best be utilized as part of a multimarker panel.

Need for multimarker testing for risk stratification \& myocardial ischemia of the dozens of biomarkers studied for ischemia and risk stratification, only the most promising have been described in this review. As troponin is the gold standard for diagnosis of AMI, most investigators have shown that the new tests provide independent and supplemental information. N evertheless, each of these tests suffers from poor specificity towards CVD . Currently, there is no single test that complements troponin. Therefore, most researchers in this area have advocated a multimarker approach.

At this time, the most logical approach is to select markers that are linked to the various pathophysiologic events known to occur in the majority of ACS cases [33]. For example, Figure4 lists the distinct events that occur and their candidate markers in patients who have STEMI and nSTEM I plaque rupture. 0 ther etiologies for AMI, such as plaque erosion or vasospasm, may require a different set of markers. The timing of blood specimens is also a critical element to the testing sequence and interpretation of multimarker testing. Figre5 shows that it may be useful to use markers for inflammation and plaque vulnerability before the onset of chest pain. When positive, they would indicate high risk for future cardiovascular events. M arkers of ischemia would havethe most value shortly after theonset of chest pain, but before there is irreversible damage. BN $P$ could be included as part of a biomarker panel, as this hormone can be released during myocardial ischemia [34]. NT-proBN P has recently been shown to be predictive of adverse events in a 


\section{Figure 5. Relationship betw een onset of disease and relative biomarker release.}

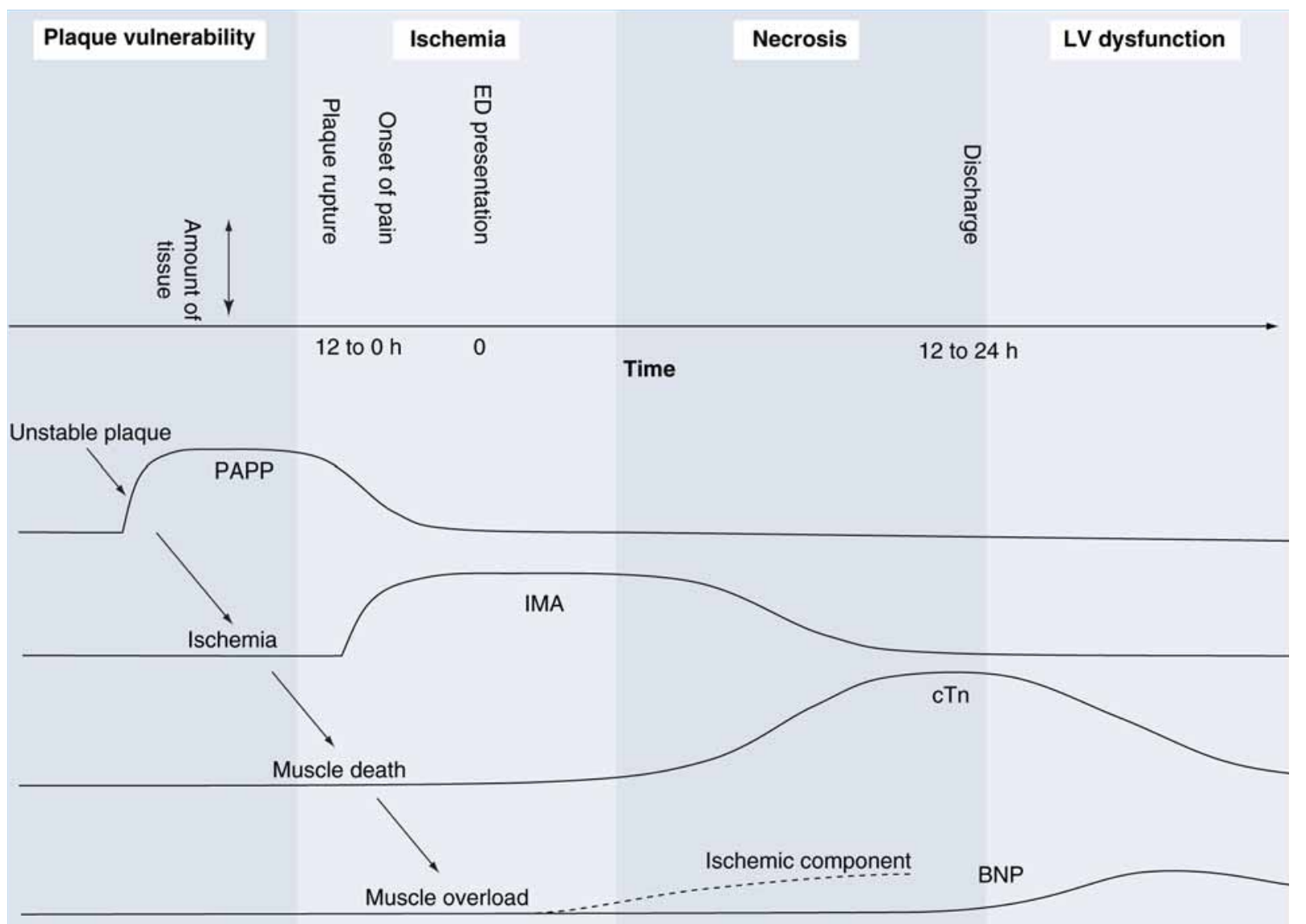

Coronary artery plaques that are vulnerable to rupture are caused by infiltration of inflammatory elements that release cytokines and enzymes that result in the degradation of the fibrous cap. PAPP-A is a metalloproteinase that is released into blood during the stage of plaque vulnerability. Once a plaque has ruptured, myocardial ischemia is the initial consequence. This can be detected by the IM A test. Prolonged ischemia leads to myocardial necrosis and release of cTn. After 12-24 h, there can be left ventricular dysfunction and myocardial muscle overloading causing the release of B-type natriuretic peptide. There may also be release of BNP due to myocardial ischemia (dotted line).

BNP: B-type natriuretic protein; CTn: Cardiac troponin; IM A: Ischemia-modified albumin; LV: Left ventricular; PAPP: Pregnancy-associated plasma protein.

group of patients with stable coronary heart disease [35]. Both markers have been shown to be largely equivalent, and therefore it is unlikely that both will be needed in a panel [36]. Troponin is most likely positive 3-9 $\mathrm{h}$ after the onset of symptoms. However, it is necessary to test troponin with every sample, as the clinical presentation is subjective, inaccurate or unknown.

The increasing interest in multivariate analysis has prompted the US FD A to issue a draft guidance document to scientists within the in vitro diagnostics industry and clinical laboratories, and the FD A staff [103]. This document defines a multivariate index as: "test systems that employ data derived in part from one or more in vitro assays, and an algorithm that usually, but not necessarily, runs on software to generate a result that diagnoses a di sease or condition, or is used in the cure, mitigation, treatment or prevention of disease."

The elements of the algorithm may include results of in vitro diagnostic assays and other laboratory data and demographic information. The algorithm assigns weights or coefficients used in calculating an index, score or classification. It is supposed that the final calculation cannot be independently derived and 
confirmed by another laboratory without access to the proprietary information used in the algorithm.

Optimization of multimarker analysis Clinical laboratory tests have traditionally relied on a cutoff concentration to differentiate between a normal and an abnormal finding. While a stepwise multivariate regression analysis can determine if the results of one marker are independent to the results of other markers for a particular disease indication, the cutoff concentrations for each test are independently optimized. A higher level of biomedical computations is necessary to determine cutoff concentrations that are optimized in the context of the other tests in the panel.

Biosite Multimarker Index ${ }^{\mathrm{TM}}$

Biosite Incorporated (CA, USA) developed a novel approach toward multimarker analysis. Figure6 plots the concentration of a biomarker against the distribution of nondiseased and diseased populations. The marker is negative in many patients who do not have the disease and positive for those who do. As with most biomarkers, there is significant overlap of biomarker concentration and the two populations. In the Multimarker Index (M MX) model, a transfer function is created for each marker. The value is either zero or one below and above the overlap region, respectively, and will be between these

\section{Figure 6. The Biosite MultiMarker ${ }^{\text {Tm }}$ Index concept.}

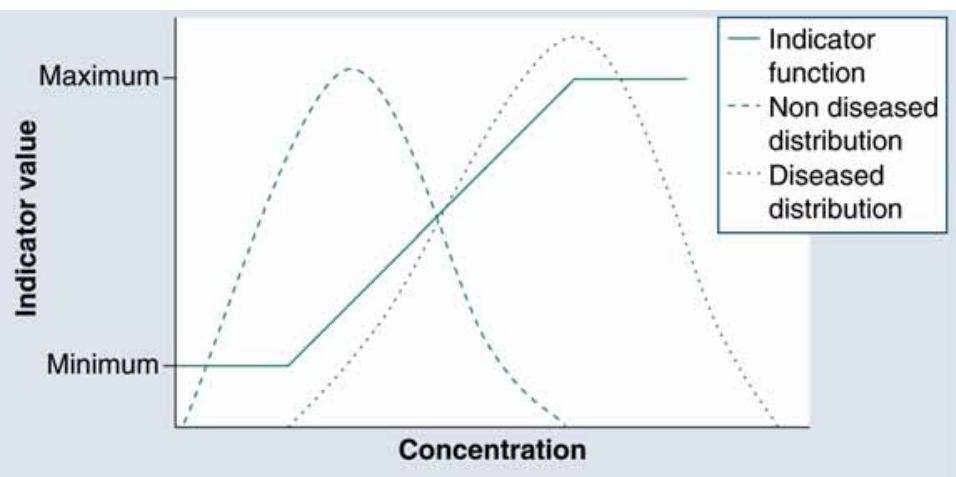

The distribution of biomarker values between patients without (left curve) and with (right curve) the disease in question, are shown. An indicator function is created in the region where there is overlap between these distributions (middle curve). The indicator function is at a minimum at the biomarker concentration at the beginning of the distribution of patients with the disease. For patients without the disease, the indicator function is a maximum at the biomarker concentration at the end of the distribution of patients without the disease. A linear function is created between these two limits. A transfer function profile is created for each biomarker in the panel. limits in a linear response for the overlap region (Figre6). The individual markers are then combined to form a multimarker index:

$$
\mathrm{MMX}=\mathrm{a}(\mathrm{Ia}[\mathrm{Ca}] \times \mathrm{Wa})
$$

Where $\mathrm{C}$ a is the concentration for analyte $\mathrm{a}$, Ia is the transfer function for analyte $a$, and has two parameters, the midpoint and range (or a low and high threshold), and $W a$ is the analyte weighting. Each biomarker has a maximum and minimum amount they can contribute with no single marker saturating the index. A search engine is used to select the parameters (midpoint, range and weighting) in the MMX. The $M M X$ cutoff value is not used during parameter optimization. The cutoff for the diagnosis of the disease in question is optimized at each facility after the parameters have been established using standard receiver operating characteristic curve analysis. The Multimarker Index has been applied to a panel of traditional markers including myoglobin, CK-M B, troponin and BN P on 2172 ED patients who presented for AM I ruleout [37]. These investigators showed that the $M M X$ was superior to the performance of the individual tests (area under the receiver operating characteristic curve: 0.98 for the M M D vs 0.78-0.94 for each marker). Work on an index for risk stratification and myocardial ischemia is in progress.

Classification \& regression trees analysis

Another approach towards multimarker analysis is the use of classification and regression trees (CART), an empirical statistical algorithm. In this model, laboratory data are organized into a flow chart where laboratory test results using optimized cut-off concentrations are progressively subdivided for groups of patients. The tree begins with one test and branches into two directions based on whether the test is positive or negative. Each daughter branch can be further subdivided based on the result of additional testing. Different tests can be applied to each daughter branch. This process continues until the regression analysis concludes that no further branching is necessary (or the number of tests in the panel has been exhausted). Regression analysis determines the optimum order of testing in the classification tree. The tree is plotted against the incidence of the particular outcome measure tested. The data do not need to be present parametrically. As an example, NT-proBNP, cT nT, serum creatinine, estimated glomerular filtration rate, CRP, hemoglobin, age and gender were 


\section{Figure 7. Classification and regression tree analysis for risk stratification of emergency department patients.}

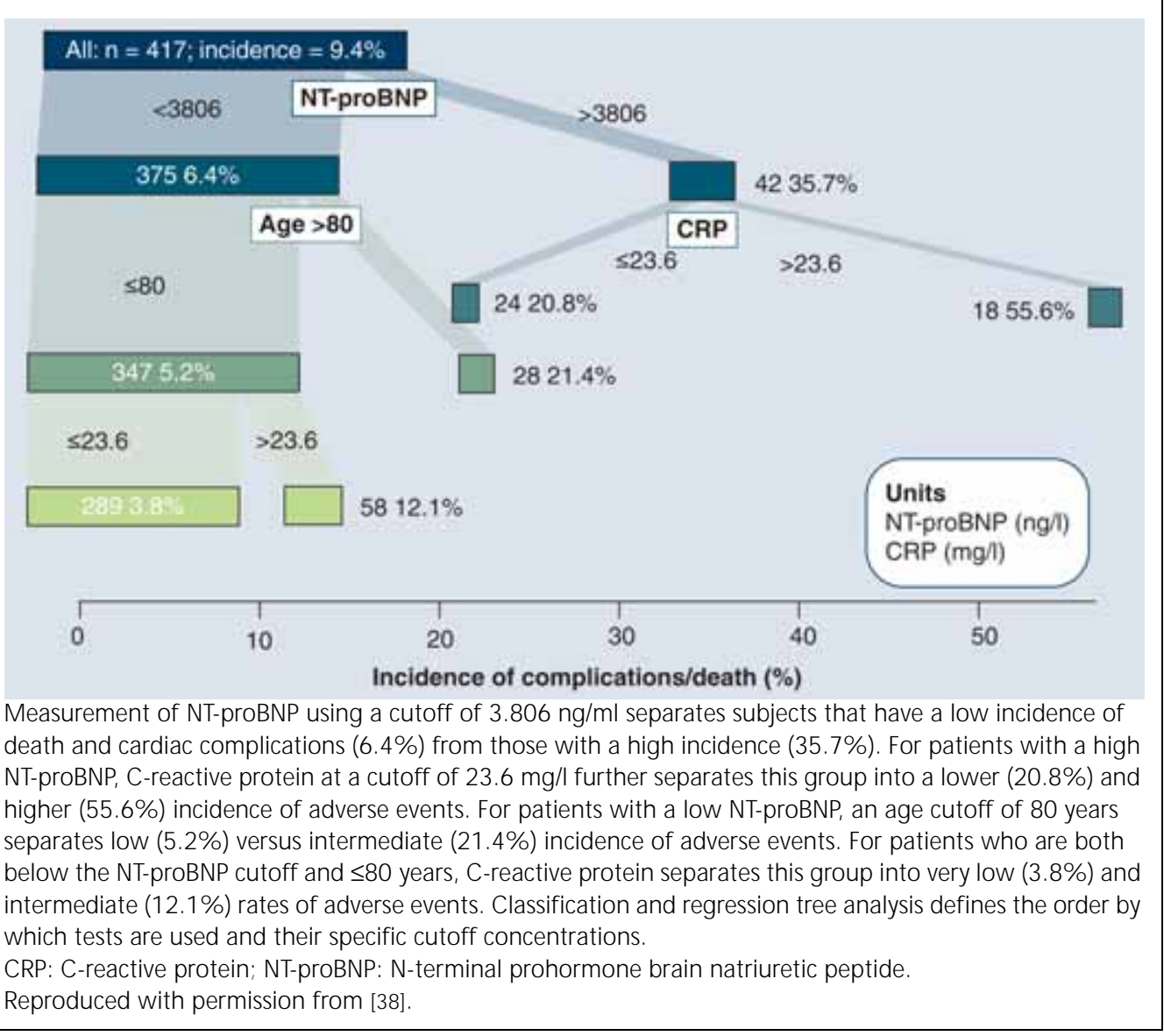

tested on ED patients for risk stratification using death and intensive care treatment as outcome measures [38]. As shown in Figure7, the tree begins with NT-proBNP testing. A normal NT -proBN P, CRP and age under 80 years was associated with the lowest incidence of complications. The highest incidence was associated with high NT-proBN P and CRP. Age was important in subdividing patients with a low N T-proBN P. The other indicators were left out of the CART model. CART analysis is ideally suited for rules for clinical decisions and can be applied for bedside analysis. Specific algorithms can be encoded into laboratory or hospital information systems that routinely report laboratory test results.

\section{Neural networks}

A neural network is a form of nonlinear artificial intelligence. The network combines data to form patterns of test results much in the same manner as the human brain is able to process light and dark shapes and colors into visual images. The network must be trained by correlating laboratory data with clinical outcomes. O nce a pattern has emerged, the network is tested by providing data and determining the accuracy of its outcome predictions. Unlike the M MX or CART, where a dichotomous cutoff concentration is established for each test independent of each other, a neural network does not use individual cut-offs. An individual test of a panel will likely have different degrees (or multiples of normal) depending on the disease entity present. The establishment of a neural network for risk stratification is shown in Figure8. The different in puts include markers that characterize a different phase of the pathophysiology of ACS. The outcome is risk or no risk, rather than the results of individual tests.

The concept of neural networks has been previously tested for biomarkers of ACS. Baxt combined 40 variables, including three laboratory tests as a network for the diagnosis of myocardial infarction [39]. The clinical sensitivity and specificity was 94.5 and $95.9 \%$, respectively. W hile these results are good, they are not 
Figure 8. Multimarker analysis of cardiac biomarkers using a neural network.

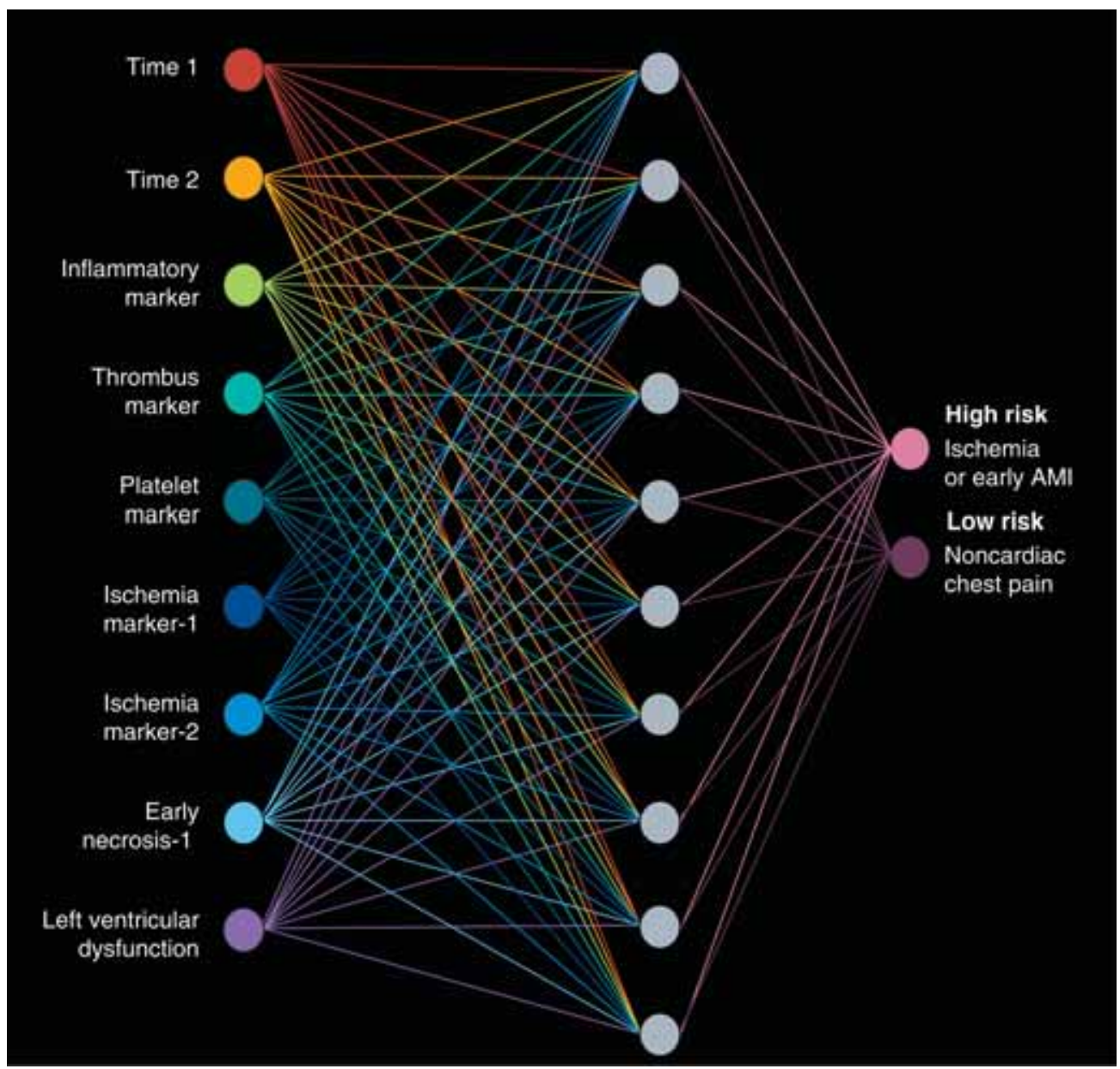

The results of different biomarkers on blood collected at one or two early time points after a patient with chest pain presents to the emergency department are inputted into the neural network program. Based on the inter-relationships between these markers and time points, the network produces a value that can be interpreted as either ischemia or early AMI, thereby putting the patient at high risk for death or MI in the short term, or the presenting symptoms are found to be consistent with noncardiac chest pain and the individual is at low-risk for adverse events. Neural networks do not separate biomarker testing results into negative and positive groups.

AMI: Acute myocardial infarction; LV: Left ventricle.

substantially better than troponin alone and may not have been the best platform to test the capabilities of the network. D etection of myocardial ischemia and risk stratification will likely be a better model to demonstrate the enhanced ability of a neural network, as there is no single test that performs as well as troponin does for AM I diagnosis.

\section{Future perspective}

The need for novel cardiac markers will continue as the population in the USA and the Western world increases. Other regions of the world, such as Asia, have also experienced an increased incidence of CVD, as their affluence has improved and their diets and exercise habits have changed such that they are at higher risk for CVDs than ever before. A multimarker approach may be needed to solve the more difficult problems such as the diagnosis of myocardial ischemia and early risk stratification for future near-term cardiovascular events. $M$ anufacturers of in vitro diagnostics equipment and experts in bioinformatics have begun developing analytical systems and interpretative al gorithms that will enable routine multimarker testing. Acceptance by clinicians, regulatory and reimbursement agencies will be decidedly slower as it represents a shift from the traditional approach towards biomarker testing. 
Executive summary

Pathophysiology of acute coronary syndromes (ACS)

- Rupture of a coronary artery is the most frequent etiology of ACS.

- Inflammation, plaque instability and myocardial ischemia are steps that preceed necrosis.

\section{Existing biomarkers for acute myocardial infarction (AMI)}

- Creatine kinase and its MB isoenzyme and myoglobin have been replaced with troponin.

- B-type natriuretic peptide and $\mathrm{N}$-terminal prohormone brain natriuretic peptide are strong predictors of heart failure and cardiovascular death.

\section{European Society of Cardiology/American College of Cardiology redefinition of AMI}

- AMI is predicated on an increase in the concentration of cardiac troponin in blood.

\section{Clinical need for early biomarkers of ACS}

- Troponin is not reliably increased in the first few hours after AMI.

- The unmet needs include a marker for early diagnosis and rule out of ACS and risk stratification for short-term morbidity and mortality.

\section{Novel biomarkers of coronary vascular disease using proteomics and metabolomics}

- Proteomics and metabolomics promises to provide new cardiac markers.

- There are limitations to the clinical implementation of new tests.

\section{Novel biomarkers based on the pathophysiology of ACS}

- Markers of inflammation and plaque instability include C-reactive protein, placental-like growth factor, myeloperoxidase and pregnancy-associated plasma protein A.

- CD40 ligand, whole-blood choline and ischemia-modified albumin indicate plaque rupture, thrombosis and myocardial ischemia.

Need for multimarker testing for risk stratification and myocardial ischemia

- A multimarker approach is needed as individual markers lack clinical specificity.

- Algorithms are necessary to interpret multimarker data.

\section{Optimization of multimarker analysis}

- The Multimarker Index ${ }^{T M}$ produces a number that acts like a cutoff for a single test.

- Classification and regression tree analysis is an algorithm that has different branch and decision limits.

- A neural network is free from the assignment of cut-off concentrations and detects patterns of laboratory test results.

Bibliography

1. Libby P, Theroux P: Pathophysiology of coronary artery disease. Circulation 111 , 3481-3488 (2005).

2. AppleFS, Wu AHB, M air J et al.: Future biomarkers for detection of ischemia and risk stratification in acute coronary syndrome. Clin. Chem. 51, 810-824, (2005).

3. Panteghini M, Gerhardt W, AppleFS, D ati F, Ravkilde J, Wu AH B: Q uality specifications for cardiac troponin assays. Clin. Chem. Lab. M ed. 39, 174-178 (2001).

4. N omenclature and criteria for diagnosis of ischemic heart disease: report of the Joint International Society and Federation of Cardiology/World H ealth $\mathrm{O}$ rganization task force on standardization of clinical nomenclature. Circulation 59, 607-609 (1979).

5. Joint European Society of C ardiology/American College of C ardiology Committee: $M$ yocardial infarction redefined - a consensus document of the joint European Society of $\mathrm{C}$ ardiology/American
College of C ardiology Committee for the redefinition of myocardial infarction. J. Am. Coll. Cardiol. 36, 959-969 (2000).

6. Storrow AB, Gibler W B: Chest pain centers: diagnosis of acute coronary syndromes. Ann. Emerg. M ed. 35, 449-461 (2000).

7. Lindahl $B$, Andren $B, O$ hlsson J, Venge $P$, Wallentin L. Risk stratification in unstable coronary artery disease. Additive value of troponin $T$ determinations and predischarge exercise tests, FRISK Study Group. Eur. H eart J. 18, 762-770 (1997).

8. Vasan RS: Biomarkers of cardiovascular disease: molecular basis and practical considerations. Circulation 113, 2335-2362 (2006).

9. Arab S, Gramolini AO, Ping P et al.: Cardiovascular proteomics. Tools to develop novel biomarkers and potential applications. J. Am. Coll. Cardiol. 48, 1733-1741 (2006).

10. Vivanco $F, M$ artin-Ventura JL, D uran M C et al.: Q uest for novel cardiovascuar biomarkers by proteomic analysis. J. Protome Res 4, 1181-1191 (2005).
11. Granger CB, Van Eyk JE, M ockrin SC, Anderson N L: N ational $\mathrm{H}$ eart, Lung, and Blood Institute Clinical Proteomics Working Group report. Circulation 109, 1697-1703 (2004).

12. M artin-VenturaJ L, D uran M C, Blanco-Colio LM et al.: Identification by a differential proteomic approach of heat shock protein 27 as a potential marker of atherosclerosis. Circulation 100, 2216-2219 (2004).

13. M arshall J, Kupchak P, Zhu W et al.: Processing of serum proteins underlies the mass spectral fingerprinting of myocardial infarction. J. Protome Res. 2, 361-372 (2003).

14. Lindsey M L, G oshorn DK, Comte-Walters $S$ et al.: A multidimensional proteomic approach to identify hypertrophy-associated proteins. Proteomics 6, 2225-2235 (2006).

15. Brindle JT, Antti H, H olmes E et al.: Rapid and noninvasive diagnosis of the presence and severity of coronary heart disease using ${ }^{1} \mathrm{H}-\mathrm{N}$ M R-based metabolonomics. $N$ ature M ed. 8, 1439-1444 (2002). 


\section{Acute coronary syndromes and risk stratification by multimarker analysis - RE V IEW}

16. Sabatine M S, Liu E, M orrow DA et al.: $M$ etabolomic identification of novel biomakers of myocardial ischemia. Circulation 112, 3868-3875 (2005).

17. Kiernan UA, N edelkov D, N elson RW: Multiplexed mass spectrometric immunoassay in biomarker research: a novel approach to the determination of a myocardial infarct. J. Proteome Res. 5 , 2928-2934 (2007).

18. Wu AH B: A selected history and future of immunoassay development and applications in clinical chemistry. Clin. Chim. Acta 369, 119-124 (2006).

19. Parikh SV, de Lemos JA: Biomarkers in cardiovascular disease: integrating pathophysiology into clinical practice. Am. J. M ed. Sci. 332, 186-197 (2006).

20. Ridker PM, Rifai N, Rose L, Buring JE, Cook N R: C omparison of $C$-reactive protein and low-density lipoprotein cholesterol levels in the prediction of first cardiovascular events. N. Engl. J. M ed. 247, 1557-1565 (2002).

21. Sabatine M S, M orrow DA, de Lemos JA et al.: M ultimarker approach to risk stratification in non-ST elevation acute coronary syndromes: simultaneous assessment of troponin I, C-reactive protein, and B-type natriuretic peptide. Circulation 105, 1760-1763 (2002).

22. H eeschen $\mathrm{C}, \mathrm{D}$ immeler $\mathrm{S}$, Fichtlscherer $\mathrm{S}$ et al.: Prognostic value of placental growth factor in patients with acute chest pain. JAM A 291, 435-441 (2004).

23. Zhang $R$, Brennan $M L$, Fu $X$ et al.: Association between myeloperoxidase levels and risk of coronary artery disease. JAM A 296, 2136-2142 (2001).

24. Baldus $S, H$ eeschen $C, M$ einertz $T$ et al.: $M$ yeloperoxidase serum levels predict risk in patients with acute coronary syndromes. Circulation 108, 1440-1448 (2003).

25. Wittfooth S, Q in Q P, Lund J et al.: Immunofluorometric point-of-care assays for the detection of acute coronary syndromerelated noncomplexed pregnancy-associated plasma protein A. Clin. Chem. 52, 1794-1801 (2006).

26. Q in Q P, Kokkala S, Lund J et al.: Immunoassays developed for pregnancyassociated plasma protein-A (PAPP-A) in pregnancy may not recognize PAPP-A in acute coronary syndromes. Clin. Chem. 52, 398-404 (2006).

27. H eeschen C, D immeler S, H amm CW, Fichtlscherer S, Simoons M L, Zeiher AM . Pregnancy-associated plasma protein-A levels in patients with acute coronary syndromes. J. Am. Coll. Cardiol. 45, 229-237 (2005).

28. Schonbeck U, Lippy P: CD 40 signaling and plaque instability. Circ. Res. 89, 1092-1103 (2001).

29. Varo N, de LemosJA, Libby P et al.: Soluble CD 40L. Risk prediction after acute coronary syndromes. Circulation 108 , 1049-1052 (2003).

30. H eeschen C, Dimmeler S, $\mathrm{H}$ amm CW et al.: Soluble CD 40 ligand in acute coronary syndromes. N . Engl. J. M ed. 248, 1104-1111 (2003).

31. Danne 0, M ockel $M$, Leuders $C$ et al.: Prognostic implications of elevated whole blood choline levels in acute coronary syndromes. Am. J. Cardiol. 91, 1060-1070 (2003).

32. Peacock F, M orris D L, Anwaruddin S et al.: $M$ eta-analysis of ischemia-modified al bumin to rule out acute coronary syndromes in the emergency department. Am. H eart J. 152, 253-262 (2006).

33. M orrow D A, Braunwald $E$ : Future of biomakers in acute coronary syndromes. M oving toward a multimarker strategy. Circulation 108, 250-252 (2003).

34. Sabatine M S, M orrow DA, de Lemos JA et al.: Acute changes in circulating natriuretic peptide levels in relation to myocardial ischemia. J. Am. Coll. Cardiol. 44, 1988-1995 (2004).
35. Bibbins-D omingo $K$, Gupta $R, N a B$, Wu AH B, Schiller N B, Whooley M A: $\mathrm{N}$-terminal pro-B-type natriuretic peptide and incident heart failure in ambulatory patients with coronary disease: data from the H eart and Soul Study. JAM A 297, 169-176 (2007).

36. Cameron $\mathrm{SJ}, \mathrm{Green} \mathrm{GB}, \mathrm{W}$ hite $\mathrm{CN}$ et al.: Assessment of BN P and NT-proBN P in emergency department patients presenting with suspected acute coronary syndromes. Clin. Biochem. 39, 11-18 (2006).

37. H ollander JE, Peacock W F, Shofer FS et al.: A cardiac multimarker index performs better than standard markers to diagnose acute myocardial infarction. Acad. Emerg. M ed. 12(5 Suppl.1), 33 (2005).

38. M ockel M, M uller R, Vollert JO et al.: Role of $\mathrm{N}$-terminal pro-B-type natriuretic peptide in risk stratification in patients presenting in the emergency room. Clin. Chem. 51, 1624-1631 (2005).

39. Baxt WG , Shofer FS, Sites FD $\mathrm{H}$ ollander JE: A neural computational aid to the diagnosis of acute myocardial infarction. Ann. Emerg. M ed. 39, 366-373 (2002).

\section{Websites}

101. H eart disease and stroke. Statistics - 2004 update. American $\mathrm{H}$ eart Association www.americanheart.org/downloadable/heart /1079736729696H D SStats2004U pdateRE V3-19-04.pdf

102. Institutional Clinical and Translational Science Award http://grants.nih.gov/grants/guide/rfafiles/RFA-RM -06-002.html

103. D raft guidance for industry, clinical laboratories, and FD A staff on in vitro diagnostic multivariate index assays www.fda.gov/cdrh/oivd/guidance/1610.pdf 